P. Lohnstein ${ }^{1,3} \cdot$ J. Schipper ${ }^{1,3} \cdot$ M. Tatagiba ${ }^{4} \cdot$ N.-C. Gellrich ${ }^{5} \cdot$ A. Berlis ${ }^{2,3} \cdot$ W. Maier ${ }^{1,3}$

${ }^{1}$ Klinik für Hals-, Nasen- und Ohrenheilkunde, Universitätsklinikum Freiburg i. Br.

${ }^{2}$ Sektion Neuroradiologie, Neurozentrum, Universitätsklinikum Freiburg i. Br.

${ }^{3}$ Interdisziplinäres Zentrum für Schädelbasis- und kraniofaziale

Chirurgie, Universitätsklinikum Freiburg i. Br.

${ }^{4}$ Neurochirurgische Universitätsklinik Tübingen

${ }^{5}$ Klinik für Mund, Kiefer-und Gesichtschirurgie, Medizinische Hochschule Hannover

\title{
Schädelbasis- chondrosarkome
}

\section{Eine interdisziplinäre Herausforderung}

Das Chondrosarkom ist einer der seltensten malignen Tumoren der Oto- und Rhinobasis: es macht nur $0,15 \%$ aller intrakraniellen Tumoren aus, wobei etwa $75 \%$ an der Schädelbasis lokalisiert sind [4]. Dementsprechend beschränken sich die meisten Publikationen auf Fallberichte oder kleine Patientenkohorten. So sind im deutschsprachigen otorhinologischen Schrifttum in den letzten 25 Jahren nur 2 Fallberichte zum Schädelbasischondrosarkom erschienen $[20,36]$. Schwerdtfeger beschrieb 1911 erstmals ein Chondrosarkom in den Nasennebenhöhlen (zit. nach [35]). Durch Ewing wurden 1939 erstmals Chondrosarkome von den Osteosarkomen histopathologisch abgegrenzt [11].

Embryologisch lässt sich ihr Auftreten an der Schädelbasis mit der dort vorherrschenden enchondralen Ossifikation erklären [22, 35]. Demgegenüber tritt an der Kalotte, an der die Ossifikation nicht enchondral abläuft, extrem selten ein Chondrosarkom auf. Besonders das Foramen lacerum, an dem die sphenopetrosale, die petrookzipitale und die sphenookzipitale Synchondrose zusammentreffen, gilt als Prädilektionsstelle $[22,26]$. Eine andere relevante Lokalisation des Chondrosarkoms im Kopf-Hals-Bereich ist der Kehlkopfknorpel [30, 37]. Systematisch wird von dem in der Literatur überwiegenden primären Chondrosarkom das seltenere sekundäre differenziert, dem die Entartung eines initial gutartigen Chondroms zugrunde liegt [26]. Selten können Chondrosarkome mit syndromalen Erkrankungen wie dem Maffucci-Syndrom und multiplen Enchondromen assoziiert sein [26].

Da die Chondrosarkome im frühen Stadium schmerzlos, langsam und verdrängend erst zu einem späteren Zeitpunkt infiltrativ wachsen, sind sie lange symptomarm und werden spät diagnostiziert $[5,35]$. Dann liegt häufig bereits ein bilaterales Wachstum mit ausgedehnten Destruktionen vor, was ein chirurgisches Vorgehen erschwert [5, 35]. Auch wurde das Chondrosarkom lange Zeit als weitgehend strahlenresistent betrachtet [19, 35]. Jedoch haben sich in den letzten Jahren die diagnostischen und therapeutischen Methoden deutlich verbessert, so beispielsweise durch die Navigationstechnologie.

Die vorliegende Arbeit beschreibt anhand der am interdisziplinären Zentrum für Schädelbasis- und kraniofaziale Chirurgie des Freiburger Universitätsklinikums behandelten Patienten Möglichkeiten und Grundsätze einer modernen interdisziplinären Therapie der kraniozervikalen Chondrosarkome.

\section{Patienten und Methoden}

Für die vorliegende Qualitätssicherungsanalyse werteten wir retrospektiv die
Krankengeschichten der 4 Patienten aus (2 Männer und 2 Frauen), die seit 1998 am Freiburger Zentrum für Schädelbasischirurgie wegen eines Chondrosarkoms behandelt wurden. Der jüngste Patient war zum Zeitpunkt der Therapie 21 Jahre, der älteste 57 Jahre alt. Bei einer Patientin handelte es sich um ein Rezidiv. Bei ihr war bereits im 18. Lebensjahr erstmals ein Chondrosarkom diagostiziert und behandelt worden. In 3 Fällen war das Chondrosarkom an der Rhinobasis, in einem Fall an der Otobasis lokalisiert.

Die Symptomatik bestand bei allen $\mathrm{Pa}$ tienten weniger als 6 Monate und war auf sensible/sensorische Symptome und unspezifische Zeichen einer Raumforderung (Schwellung, Obstruktion) beschränkt. Bei keinem Patienten traten motorische Störungen oder Blutungen auf. Bei allen Patienten nahmen wir ein Staging auf lokoregionäre Metastasen (Halslymphknoten) und Fernmetastasen vor. In allen Fällen wurde die diagnostische und therapeutische Strategie in der interdisziplinären Schädelbasiskonferenz erörtert und gemeinsam die Therapieempfehlung an die Patienten festgelegt.

\section{Ergebnisse}

Die mittlere Nachbeobachtungszeit seit der erstmaligen Diagnosestellung liegt bei 74 Monaten. Das Minimum beträgt 16, das 


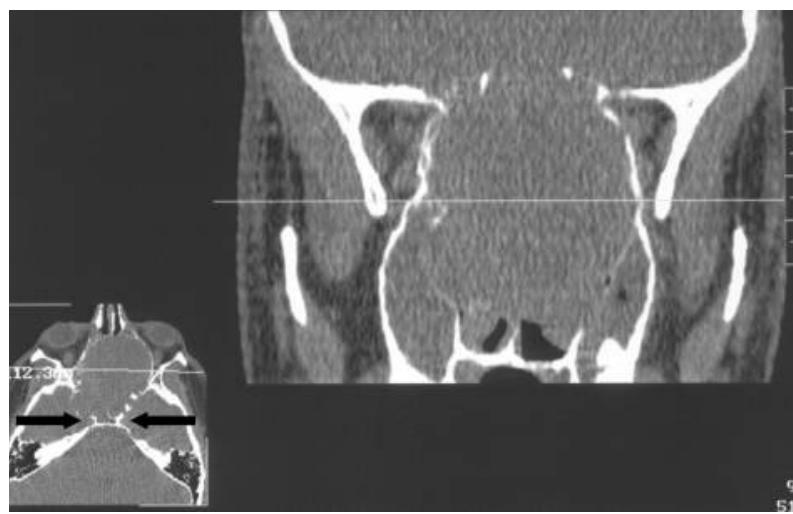

Abb. $1<$ Fall 1. 21-jähriger Mann mit median betontem rhinobasalem Chondrosarkom. Das axiale und koronare CT zeigt die knöcherne Destruktion der Rhinobasis. Der Tumor reicht beidseits bis an die A. carotis interna im Bereich des Foramen lacerum internum (Pfeile)

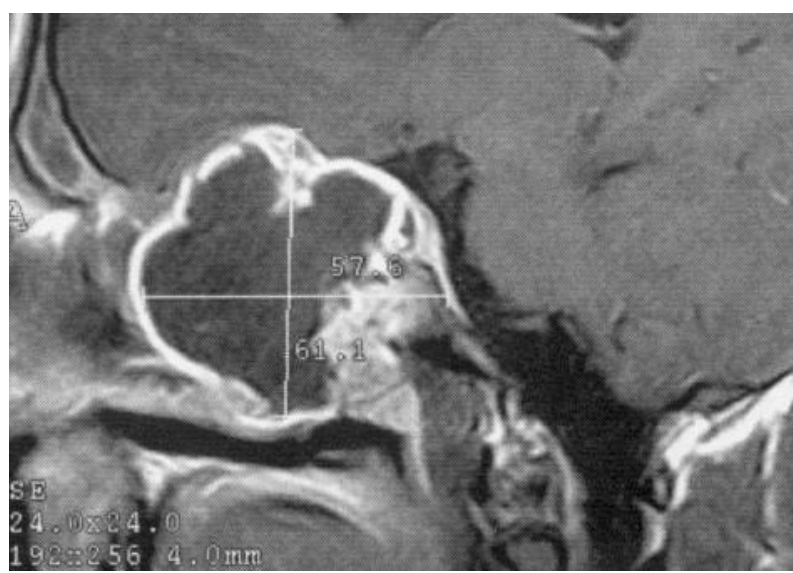

Abb. $2<$ Fall 1. Im MRT ist der Tumor in der sagittalen T1-Wichtung hypointens mit randständiger Kontrastmittelanreicherung

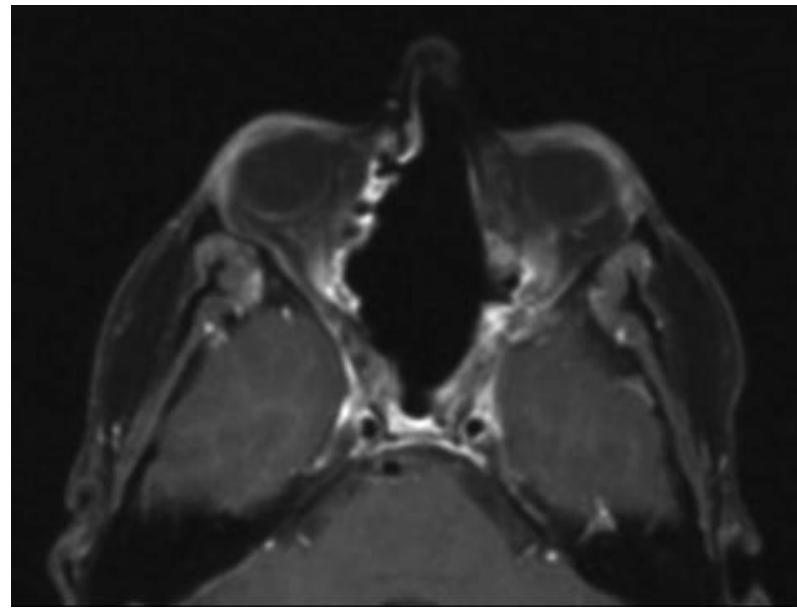

Abb. $3<$ Fall 1. 18 Monate nach Diagnosestellung und 15 Monate nach der Nachbestrahlung besteht MRtomographisch kein Anhalt auf ein Rezidiv (axiale fettunterdrückte T1-gewichtete Schicht mit Kontrastmittel)

Maximum 198 Monate (Letzteres bei der Patientin, bei der wir das Rezidivsarkom behandelten). Die Nachbeobachtungszeit seit Durchführung unserer Therapie beträgt 31 Monate (Minimum 15, Maximum 44 Monate). Bei keinem Patienten trat seither ein Rezidiv auf. Auch wurden bei keinem Patienten jemals im Verlauf des diagnostischen Stagings oder der Nachuntersuchung eine lokoregionäre Metastasierung oder Fernmetastasen beobachtet. So waren alle Patienten zum Zeitpunkt der letzten Vorstellung residuum- und re- zidivfrei, was stets durch Bildgebung, bei einer Patientin auch bioptisch, gesichert wurde. Somit errechnet sich eine mittlere Befundkonstanz und Tumorfreiheit von 31 Monaten seit Therapie.

Die anhand unserer Analyse evaluierten charakteristischen Befunde und Verlaufsformen beim Schädelbasischondrosarkom sollen im Folgenden dargestellt werden:

Fall 1 Bei dem 21-jährigen männlichen Patienten entwickelten sich über 4 Mo- nate langsam eine behinderte Nasenatmung und ein linksseitiger Exophthalmus ohne Doppelbilder. Endonasal bestand bei intakter Schleimhaut eine kranial und dorsal gelegene, das Nasenseptum auftreibende Raumforderung. Das CT der Nebenhöhlen zeigte einen ca. $6 \mathrm{~cm}$ großen Tumor, der bilateral bis an den Karotis-kanal heranreichte und partiell die knöcherne Rhinobasis destruierte (• Abb. 1). Im MRT war der Tumor in der T1-Wichtung zentral hypointens mit randständiger Kontrastmittelaufnahme, in der T2-Wichtung zentral hyperintens (- Abb. 2).

Die transnasale Biopsie sicherte die Verdachtsdiagnose eines Chondrosarkoms, das histopathologische Grading ergab einen Differenzierungsgrad 2. Wir resezierten den Tumor in einem interdisziplinären rhinoneurochirurgischen Eingriff über einen paranasalen, transfazialen $\mathrm{Zu}$ gangsweg, wobei im Bereich des tumorinfiltrierten linken Karotiskanals eine RoResektion nicht sicher möglich war. Ansonsten war die Tumorresektion vollständig. Das innere Durablatt war nicht infiltriert, sodass eine komplette Duraresektion nicht erforderlich war. Auch die Periorbita war nicht betroffen und wurde belassen.

Postoperativ bestanden keine Doppelbilder. Aufgrund der fraglichen Ro-Situation wurde postoperativ eine intensitätsmodulierte stereotaktische Photonentherapie mit 66 Gy vorgenommen. 15 Monate nach der Bestrahlung bestehen radiologisch keine Hinweise auf ein Lokal- oder lokoregionäres Rezidiv (• Abb. 3).

Fall 2 Der zum Zeitpunkt der Vorstellung 32-jährigen Patientin war seit einigen Wochen eine langsam progrediente rechtsseitige Protrusio bulbi ohne Doppelbilder aufgefallen. Visus und Motilität waren unauffällig. 14 Jahre zuvor war bei ihr ein Chondrosarkom der Rhinobasis zweizeitig reseziert und mit einer Chemotherapie nach der Deutschen Kooperativen Weichteilstudie behandelt worden. Eine Nachbestrahlung war von der Patientin abgelehnt worden.

Endonasal zeigte sich ein von glatter Schleimhaut überzogener rechtsbetonter, der Rhinobasis und der rechten Orbita breitbasig aufsitzender Tumor. Im CT 
zeigte dieser ausgeprägte Kalzifizierungsherde und im T1-gewichteten MRT eine deutliche Kontrastmittelanreicherung. Es bestand der Verdacht auf eine Orbita- und Durainfiltration. Vorgeschichte und Bildgebung wiesen auf ein Rezidiv des Chondrosarkoms hin, sodass auf eine Biopsie verzichtet wurde. Wir resezierten den Tumor in einem interdiszipliären navigationsgestützten neurorhinochirurgischen Eingriff transfrontal komplett und visuserhaltend. Die rechtsseitige mediale Periorbita musste ebenso wie Teile der Dura reseziert werden, wir rekonstruierten die rechte mediale Orbitawand und die Rhinobasis mittels intraoperativ navigationskontrolliert modelliertem Titan-Mesh und Galea-Periost.

Histologisch ergab sich ein Chondrosarkom (Grad II). Eine postoperative Bestrahlung wurde von der Patientin erneut abgelehnt. 30 Monate postoperativ (und 16 Jahre nach Erstdiagnose) ist sie rezidiv- und beschwerdefrei, es bestehen keinerlei Sehstörungen.

Fall 3 Die 24-jährige Patientin litt seit knapp 6 Monaten unter zunehmenden Zahn- und Oberkieferschmerzen rechts, die zuletzt mit einer Hypästhesie infraorbital rechts, einer rechtsseitig behinderten Nasenatmung und einem zunehmenden Geruchsverlust assoziiert waren. Bei der Erstvorstellung war die rechte Nasenhaupthöhle subtotal verlegt, Visus und Augenmotilität waren unauffällig. Im Nebenhöhlen-CT fand sich ein knochendestruierender Tumor mit kalkdichten Herden und breitflächiger Jochbein- und Orbitainfiltration ( $\bullet$ Abb. 4), im MRT eine randständige deutliche Kontrastmittelaufnahme bei zentraler Hypointenistät in der T1-Wichtung.

Die transnasale Biopsie bestätigte die Verdachtsdiagnose eines Chondrosarkoms (Grad II), welches wir interdisziplinär rhinokieferchirurgisch navigationsgestützt unter Mitnahme der kaudalen, der medialen und von Teilen der lateralen Orbitawand resezierten. In derselben Sitzung erfolgte die navigierte Mittelgesichts- und Orbitarekonstruktion mit Titanplatten und Mesh (• Abb. 5). Aufgrund der Tumorausdehnung und Infiltration war am Klivus eine Ro-Resektion nicht oder nur fraglich anzunehmen, sodass eine Pho-

P. Lohnstein · J. Schipper · M. Tatagiba · N.-C. Gellrich · A. Berlis · W. Maier Schädelbasischondrosarkome. Eine interdisziplinäre Herausforderung

Zusammenfassung

Hintergrund. Das Chondrosarkom ist eine seltene Differenzialdiagnose maligner Tumoren der Schädelbasis. Die Prognose wurde im Schrifttum lange Zeit als ungünstig beurteilt. Sie hat sich in den letzten Jahren jedoch deutlich gebessert. Ziel der Untersuchung war die Evaluation und Darstellung neuer optimierter Behandlungsstrategien.

Patienten und Methoden. Wir analysierten retrospektiv Krankengeschichte und Verlauf von 4 Patienten, die wir am Freiburger Schädelbasiszentrum in den letzten 5 Jahren wegen eines Chondrosarkoms der Schädelbasis behandelten.

Ergebnisse. Aufgrund der anfänglich geringen Symptomatik stellten sich die Patienten erst in einem fortgeschrittenen Tumorstadium vor. Alle Patienten wurden operativ versorgt, wobei eine R0-Resektion knapp oder nur fraglich vorlag. In 3 Fällen erfolgte ei-

\section{Skull base chondrosarcoma. An interdisciplinary challenge}

\author{
Abstract \\ Background. Chondrosarcoma is a rare dif- \\ ferential diagnosis of malignant tumors of \\ the skull base. The prognosis was rated as un- \\ favourable in articles for many years. It has, \\ however, improved considerably in recent \\ years. The objective of this study was to eval- \\ uate and current, new optimised treatment \\ strategies. \\ Patients and Methods. We retrospectively \\ analysed the case histories and course of four \\ patients whom we treated for chondrosarco- \\ ma of the skull base over the past 5 years at \\ the Freiburg Skull Base Centre. \\ Results. Because of initially mild symp- \\ toms, the patients first came for examination \\ at an advanced stage of the tumor. All pa- \\ tients underwent surgery, whereby an R0-re- \\ section was barely or only questionably pres-
}

ne Nachbestrahlung. Bis zum heutigen Zeitpunkt sind alle Patienten tumorfrei. Schlussfolgerungen. Die operative Sanierung mit kurativer Intention ist grundsätzlich Therapie der Wahl. Aufgrund der meist großen Tumorausdehnung und engen Beziehung zu relevanten Strukturen ist trotz der Fortentwicklungen operativer Verfahren eine vollständige Resektion nicht immer möglich. Unter Berücksichtigung der Möglichkeiten moderner adjuvanter strahlentherapeutischer Verfahren wird einer inkompletten funktionserhaltenden Resektion der Vorzug gegenüber einer radikalen und mutilisierenden Resektion gegeben.

\section{Schlüsselwörter}

Chondrosarkom · Klivus · Computerassistierte Chirurgie $\cdot$ Nasennebenhöhlen .

Schädelbasistumoren ent. Three patients underwent radiation therapy postoperatively. All patients are currently tumor free.

Conclusions. Surgical treatment with curative intent is basically the therapy of choice. Due to the usually large size of the tumor and its close relationship to relevant structures, complete resection is, however, not always possible despite advances in surgical procedures. Taking the possibility of modern adjuvant radiotherapeutic procedures into account, an incomplete, function-preserving resection is preferred to a radical and mutilating resection.

\section{Keywords}

Chondrosarcoma · Clivus · Computer assisted surgery · Paranasal sinuses - Skull base tumors 


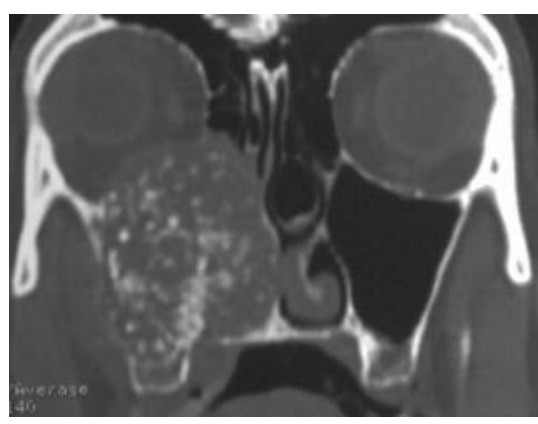

Abb. $4 \Delta$ Fall 3. 24-jährige Patientin mit Osteosarkom der rechten Kieferhöhle. Im CT ist die Infiltration von Orbitainhalt und Jochbein erkennbar. Die popcornartigen Verkalkungen und die paramediane Lage sind typisch für ein Chondrosarkom

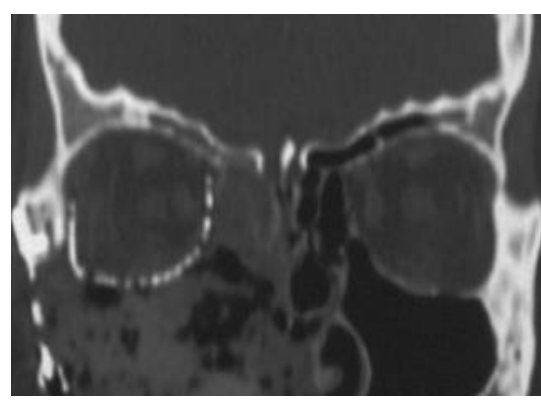

Abb. $5 \Delta$ Fall 3. Das postoperative CT dokumentiert die exakte Lage der navigationsgestützt mit Titanmesh rekonstruierten Orbitawände (wie in $\bullet$ Abb. 4 handelt es sich um eine koronare Sekundärrekonstruktion eines 3D-CT-Datensatzes)

tonentherapie mit einem Zielvolumen in den dorsalen Kieferhöhlenanteilen unter Einschluss der retromaxillären Strukturen bis zum Klivus und der Fossa infratemporalis angeschlossen wurde.

Wegen eines einige Monate später auftretenden perforierenden Weichteildefektes mussten wir die Implantate teilweise entfernen und den Defekt mit einem M.-latissimus-dorsi-Lappen decken, wodurch auch der Gaumendefekt verschlossen wurde. Bildgebende Untersuchungen und mehrfache Probebiopsien ergaben bis heute (44 Monate nach Diagnosestellung) keine Hinweise auf ein Tumorrezidiv.

Fall 4 Bei dem 57-jährigen Patienten trat über wenige Monate eine langsam progrediente Schwellung im Bereich der rechten Kiefergelenkes auf, die sich bioptisch als Chondrosarkom (Grad I) erwies. Die Bildgebung zeigte einen vom Kiefergelenk ausgehend in die Fossa temporalis einwachsenden und die Dura infiltrierenden Tumor. Dieser wurde navigationsgestützt unter Erhalt des N. facialis und mit Resektion des Kiefergelenkes und von Teilen des M. temporalis und der laterobasalen Dura sowie der vorderen Gehörgangswand in toto exstirpiert. Darüber hinaus erfolgte eine Duraplastik, und der Gehörgang wurde mit Knorpel rekonstruiert. Der postoperative Verlauf war unauffällig. Aufgrund der knappen Ro-Resektion zum Temorallappen wurde eine kleinvolumige fraktionierte Radiotherapie angeschlossen. 36 Monate postoperativ ist der Patient rezidiv- und beschwerdefrei.

\section{Diskussion}

\section{Histologische Klassifikation}

Chondrosarkome werden entsprechend ihren histologischen Malignitätskriterien (Mitoseindex, Kernpolymorphien, Zellreichtum) in 3 Subtypen unterteilt [10]. An der Schädelbasis überwiegen die niedrig malignen Formen des Chondrosarkoms (Grad I und II), die in dieser Region über 99\% aller Fälle ausmachen [28]. Da die niedrig malignen Chondrosarkome langsam und zunächst nur verdrängend wachsen, sind die Symptome - wie auch bei unseren Patienten - lange Zeit gering. Vom Grad III wird das mesenchymale Chondrosarkom als separate, besonders maligne Entität abgegrenzt, die sich durch histologischen Nachweis primitiver Spindelzellen, verbunden mit rascher Durainfiltration auszeichnet $[10,19]$. Die Prognose scheint mit der Graduierung zu korrelieren [10]. Metastasen treten bei Chondrosarkomen der Schädelbasis in weniger als $10 \%$ der Fälle auf $[5,16]$ und wurden bei Tumoren vom Grad I bisher nicht beobachtet [10].

Die Abgrenzung zu anderen Läsionen der Schädelbasis, insbesondere zum Chordom, war bis zur Etablierung immunhistochemischer Methoden schwierig. Dementsprechend subsumieren viele, insbesondere ältere Arbeiten beide Krankheiten, was die Zahl hinsichtlich Verlauf und Prognose aussagekräftiger Publikationen reduziert. Im Unterschied zu Chordomen exprimieren Chondrosarkome immunhistochemisch Vimentin. Hingegen fehlt die Expression epithelialer Marker wie Zytokeratin und EMA [19, 33, 35]. So wird die Einordnung der sog. „chon- droiden Chordome“ noch in der neuesten Literatur kontrovers diskutiert: während einige Autoren diese als niedrig maligne Chondrosarkome ansehen [33], werden sie von anderen als eine eine Untergruppe der Chordome klassifiziert [22].

\section{Einsatz bildgebender Verfahren}

Die Bildgebung erlaubt in vielen Fällen die Verdachtsdiagnose, welche bei guter $\mathrm{Zu}$ gänglichkeit des Tumors im Regelfall vor Entscheidung über das therapeutische Vorgehen bioptisch gesichert werden sollte. Im CT imponiert das Chondrosarkom als unregelmäßig begrenzte osteodestruktive Raumforderung, die typischerweise (aber nicht zwingend) von multiplen Kalzifikationen durchsetzt ist, dem sog. Popcornmuster [26, 35]. Da diese Tumoren an der Schädelbasis vornehmlich von der Synchondrose entlang des Foramen lacerum ausgehen, sind sie im Gegensatz zu den median entstehenden Chordomen meist paramedian gelegen und lassen sich dadurch von diesen differenzieren. Dies ist bei unseren Patienten insbesondere im 3., aber auch im 2. Fall gegeben.

Ein Fehlen der Kalzifikationen schließt ein Chondrosarkom allerdings nicht aus. Die MRT-Befunde sind im Vergleich zu den typischen CT-Befunden weniger wegweisend, wenngleich die Vorteile v. a. in Weichgewebsbeurteilung der peritumoralen Strukturen liegen. Weiterhin ermöglicht die MR-Angiographie eine Gefäßbeurteilung, insbesondere auch im Hinblick auf die Lagebeziehung des Tumors zur A. carotis interna. Chondrosarkome weisen häufig eine geringe und inhomogene Kontrastmittelaufnahme auf. Insbesondere zentrale Hypointensität in der T1-gewichteten Darstellung mit randständiger Kontrastmittelanreicherung sowie zentrale Hyperintensität in der T2Wichtung sind beschrieben worden [23], und waren bei unseren Patienten 1 und 2 ebenfalls nachzuweisen.

\section{Entwicklung und Ergebnisse chirurgischer Therapieverfahren}

Da im Schädelbasisbereich kurative Tumorresektionen selten möglich waren [12], wurde initial ein Tumordebulking als Therapie der Wahl angesehen, das jedoch 
bei der überwiegenden Mehrzahl der Patienten mit rascher Progredienz des $\mathrm{Re}$ sidualtumors verbunden war $[19,21,26]$. Der konventionellen Strahlentherapie wird im Schrifttum nur begrenzter Wert beigemessen $[6,10]$ : ohne vorangehende sanierende Operation sprach (allerdings bei starker Variationsbreite je nach Differenzierungsgrad des Tumors) der Tumor in etwa der Hälfte der Fälle an [15].

Harwood beobachtete eine komplette Remission bei weniger als einem Viertel seiner Patienten [15], und Coates erzielte bei Rezidiven keine kurativen Erfolge durch Bestrahlung [5]. Dies ist weitgehend durch die unmittelbare Nachbarschaft strahlensensibler funktionell oder vital bedeutender Organstrukturen und dadurch bedingte geringere Dosis begründet und nicht durch eine grundsätzliche Strahlenresistenz der Chondrosarkome $[15,32,35]$. So wird insbesondere in der älteren Literatur die Prognose des Schädelbasischondrosarkoms meist als ungünstig beschrieben $[5,6,12,26]$.

Burkey beschrieb in seiner Patientenserie bei Chondrosarkomen der $\mathrm{Na}$ se oder Nebenhöhlen ein Überleben nach 5 Jahren von etwa 60\% [2]. Demgegenüber wurde bereits 1977 für die Gesamtheit der Chondrosarkome, also aller Lokalisationen des Körpers, eine 5-Jahres-Überlebensrate für den Grad I von 90\%, für Grad II von etwa $80 \%$ und nur für Grad III von unter 50\% beschrieben, ermöglicht durch eine radikale Tumorresektion [10].

Ziel einer Weiterentwicklung der Chondrosarkomtherapie im Schädelbasisbereich war daher stets, eine komplette Resektion möglichst in einem Blockpräparat zu erreichen $[21,34,35]$, auch um eine Tumorverschleppung sowie ein erhöhtes Risiko durch Liquorrhö oder Verletzung von benachbarten Strukturen bei wiederholten Eingriffen zu vermeiden. Jedoch erst vor etwa 20 Jahren wurden erstmals Fälle kompletter Resektion eines Schädelbasischondrosarkoms beschrieben $[13,29]$ ermöglicht durch eine Fortentwicklung der chirurgischen Techniken [35].

In der letzten Dekade hat auch der Einsatz der Navigation sowie das intraoperative Neuromonitoring hierzu in hohem Maße beigetragen, da diese es dem erfahrenen Operateur erlauben, die intraoperative Präparation mit erhöhter Sicherheit besonders gegenüber kritischen Nachbarstrukturen vorzunehmen [31]. Aufgrund des meist primär extraduralen Tumorwachstums ist eine vollständige Resektion grundsätzlich erreichbar, wenn auch das infiltrative Wachstum eine Ro-Resektion häufig unmöglich macht [35], insbesondere bei Infiltration vital oder funktionell relevanter Strukturen [35]. In diesen Fällen gilt es zwischen einer radikalen Resektion mit potenziell mutilierenden $\mathrm{Ne}$ benwirkungen und einer funktionserhaltenden Resektion abzuwägen.

So kann die Resektion der A. carotis interna $[13,29]$ mit einer Hemiparese einhergehen - ein Risiko, das auch durch einen vorangegangenen Karotisokklusionsversuch nicht vollständig ausgeschlossen werden kann. Der Verschluss des aus der Schädelbasisoperation resultierenden Defektes kann - insbesondere bei Beteiligung der Rhinobasis - problematisch sein. Bereits präoperativ muss geplant werden, wie der Defekt geschlossen werden soll. Bei größeren Defekten ist ein Hartgewebsersatz erforderlich, um eine Herniation von intrakraniellem oder orbitalem Gewebe zu vermeiden. Hierzu kann entweder körpereigenes Knochenmaterial [38] oder ein alloplastisches Transplantat verwendet werden [7]. Bei unserer 2. Patientin haben wir erfolgreich ein Titanimplantat eingesetzt, das intraoperativ navigationsgestützt modelliert wurde, um die Basis und die Orbita funktionell optimal zu rekonstruieren.

In jedem Falle ist jedoch der Weichgewebsverschluss vorzunehmen, der zur Vermeidung einer Liquorfistel aus vitalem durchblutetem Gewebe bestehen sollte [38].

\section{Stellenwert radiothera- peutischer Verfahren}

Das neuere Schrifttum empfiehlt vermehrt, mutilierende Eingriffe zu vermeiden [35] und funktionserhaltend zu operieren, zumal sich aufgrund der Fortschritte in der Strahlenheilkunde die Möglichkeiten einer nebenwirkungsarmen und zugleich effektiven Bestrahlung residualer Tumoranteile in der Nähe sensitiver Strukturen deutlich verbessert haben $[3,8]$. Castro beschreibt eine Reduktion strahlenbedingter Komplikationen von über 40\% im Zeitraum vor 1986 auf 20\% in den anschließenden Jahren. Dies führt er auf verbesserte Techniken der Bildgebung und Planung zurück [3].

So ist die intensitätsmodulierte Photonentherapie eine Technik, bei der mit mehreren Feldern und Unterfeldern eine hohe Konzentration der Strahlendosis auf das eigentliche Zielvolumen, verbunden mit minimaler Belastung benachbarter Strukturen, erreicht wird $[8,27]$. Entsprechend wurde bei unserem 1. Patienten bei nicht sicherer Ro-Resektion im Bereich des infiltrierten Karotiskanals eine intensitätsmodulierte Photonentherapie erfolgreich angeschlossen.

Aber auch neue Formen der Bestrahlung mit Protonen oder Schwerionen [17, 27, 32] erlauben eine gezielte Radiatio auch in der unmittelbaren Nachbarschaft sensibler Strukturen wie dem Hirnstamm oder dem N. opticus. Die Reichweite der schweren Teilchen ist durch ihre physikalischen Eigenschaften definiert und durch den Bragg-Peak als Bereich der maximalen Strahlenabsorption limitiert [32], was mit einer Schonung der vor und hinter dem Bestrahlungsziel liegenden Gewebe einhergeht. So wird diese Therapie in der Regel gut und ohne schwerwiegende Nebenwirkungen toleriert [32].

Die vorteilhaften Eigenschaften moderner Bestrahlungsformen und -techniken gehen daher auch mit der Möglichkeit einher, im Einzelfall eine höhere Strahlendosis zu applizieren oder die Bestrahlung zu wiederholen [32], was im Bereich der Schädelbasis bisher nur selten möglich war. In einzelnen aktuellen Studien wurden 5-Jahres-Überlebensraten von $76-99 \%$ beschrieben $[1,28]$, wobei die Patienten inhomogene Kollektive bildeten, die sich teilweise Voropera- 
tionen unterschiedlicher Radikalität unterzogen hatten. So ist die Prognose der Chondrosarkome besser als die der Chordome, von denen jeder Fall histologisch abgegrenzt werden muss [8, 28]. Sie korreliert mit dem postoperativ verbliebenen Tumorvolumen [17].

Die neuartigen Bestrahlungsmethoden werden nur an wenigen spezialisierten Zentren und im Regelfall im Rahmen von Studien durchgeführt. Grundsätzlich ist die Strahlentherapie mit schweren Partikeln auch als primäre Bestrahlung möglich. Jedoch wird auch in der neuesten Literatur empfohlen, eine wenn möglich totale oder subtotale Tumorresektion voranzustellen [14, 19]. Die alleinige Bestrahlung wird bislang nur bei lokal oder durch den Allgemeinzustand bedingter Inoperabilität empfohlen [14].

Das Schrifttum ist in der Frage der Notwendigkeit einer postoperativen Nachbestrahlung nicht einheitlich [35]. Es muss stets die potenzielle strahlenbedingte Morbidität gegen das Risiko einer erneuten Wachstumstendenz bei bestehendem Tumorresiduum abgewogen werden. Auch ist das Risiko einer Nachbestrahlung mit zunehmender Anzahl vorangegangener chirurgischer Eingriffe erhöht [17]. Bei inkompletter Resektion wird eine Radiatio allerdings empfohlen [32].

Bei unseren Patienten lag in 2 Fällen eine nur fragliche Ro-Resektion vor. Die beiden anderen Patienten waren Ro-reseziert, allerdings handelte es sich im einen Fall um eine sehr knappe Resektion, im anderen Fall um ein Rezidivsarkom. In beiden Fällen empfahlen wir die Nachbestrahlung, die jedoch von der letztgenannten Patientin abgelehnt wurde.

In einem von 3 Fällen kam es zu einer schwerwiegenden strahlenbedingten Komplikation, die allerdings nicht im basisnahen Bereich auftrat, sondern in einer Extrusion der intraoperativ zur Rekonstruktion eingebrachten Titanplatten bestand, die daraufhin entfernt werden mussten. Dies erforderte mehrere Eingriffe zur erneuten Rekonstruktion des Mittelgesichtes und der Orbita. Dieser Fall unterstreicht die Notwendigkeit einer Abwägung aller für und gegen eine Bestrahlung sprechenden Fakten. Somit kann das Einbringen von alloplastischem Material im Rahmen der Rekons- truktion mit einem Risiko der Extrusion und somit mit erhöhter Strahlenmorbidität einhergehen [9]. Dieses Risiko kann allerdings vermindert werden, wenn primär der resektionsbedingte Hohlraum im Mittelgesicht durch ein Weichgewebstransplantat, beispielsweise vom M. latissimus dorsi, obliteriert und zugleich das Implantat auch zur Haut hin mit ausreichend körpereigenem Weichgewebe unterfüttert wird [25].

Über stereotaktische Verfahren mit einmaliger Hochdosisbestrahlung liegen nur wenige Berichte mit sehr geringen Fallzahlen vor. Sie finden in einzelnen Zentren bei kleinen Zielvolumina Anwendung und scheinen mit geringen Nebenwirkungen assoziiert zu sein [24].

\section{Chemotherapie}

Im Schrifttum gibt es keine überzeugenden Daten über einen erfolgreichen Einsatz der Chemotherapie [2, 18, 35], jedoch wurden insbesondere in der 1980erJahren Protokolle wie bei Weichteilsarkomen in Einzelfällen erfolgreich eingesetzt [12]. So bestand auch bei unserer 2. Patientin ein über 10 Jahre andauerndes freies Intervall nach radikaler Operation und Chemotherapie nach CWS-Schema, wobei allerdings die Bedeutung der Chemotherapie für den Verlauf offen bleiben muss. Insbesondere bei hochmalignen Formen des Chondrosarkoms, die an der Schädelbasis selten auftreten, wird die Chemotherapie im Schrifttum diskutiert [18]. Unseres Wissens liegt aufgrund der geringen Patientenzahl bislang keine prospektive Studie zu dieser Fragestellung vor [18].

Bei Auftreten eines Rezidivs sollte im Einzelfall abgewogen werden, ob dieses erneut operiert oder strahlentherapeutisch angegangen wird. In der Entscheidungsfindung sollten die Lokalisation des Rezidivs, Alter und Allgemeinzustand des Patienten sowie die individuelle spezifische Krankheitsvorgeschichte einfließen.

\section{Fazit für die Praxis}

\section{Bei Schädelbasischondrosarkomen ist eine interdisziplinäre Planung der dia- gnostischen und therapeutischen Maß-}

nahmen von Anbeginn angezeigt. Die Prognose dieser seltenen Erkrankung hat sich in den letzten Jahren, bedingt durch Fortschritte sowohl im operativen als auch im strahlentherapeutischen Sektor erheblich verbessert. Der Patient sollte daher frühzeitig in einem spezialisierten Zentrum vorgestellt werden. Durch ein interdisziplinäres Vorgehen mit modernen Methoden kann in vielen Fällen ein langjähriges Überleben mit guter Lebensqualität erreicht werden.

\section{Korrespondierender Autor}

\section{P. Lohnstein}

Universitäts-HNO-Klinik

Killianstraße 5, 79106 Freiburg

lohnstein@hno1.ukl.uni-freiburg.de

Interessenkonflikt. Keine Angaben

\section{Literatur}

1. Austin-Seymour M, Munzenrider J, Goitein M et al (1989) Fractionated proton radiation therapy of chordoma and low-grade chondrosarcoma of the base of the skull. J Neurosurg 70: 13-17

2. Burkey BB, Hoffman HT, Baker SR, Thornton AF, McClatchey KD (1990) Chondrosarcoma of the head and neck. Laryngoscope 100: 1301-1305

3. Castro JR, Linstadt DE, Bahary JP et al. (1994) Experience in charged particle irradiation of tumors of the skull base: 1977-1992. Int J Rad Oncol Biol Phys 29: 647-655

4. Cianfriglia F, Pompili A, Occhipinti E (1978) Intracranial malignant cartilaginous tumours. Report of two cases and review of literature. Acta Neurochir 45: 163-175

5. Coates HL, Pearson BW, Devine KD, Unni KK (1977) Chondrosarcoma of the nasal cavity, paranasal sinuses, and nasopharynx. Transactions Otolaryngol 84: 919-926

6. Coltrera MD, Googe PB, Harrist TJ, Hyams VJ, Schiller AL, Goodman ML (1986) Chondrosarcoma of the temporal bone. Diagnosis and treatment of 13 cases and review of the literature. Cancer 58: 2689-2696

7. Dacho A, Steffen H, Ziegler CM, Conradt C, Dietz A (2002) Orbitabodenrekonstruktion. Vergleich einer neuen perforierten $0,15 \mathrm{~mm}$ dicken PDS-Folie mit etabliertem Titanium-Dynamic-Mesh. HNO 50: 2128

8. Debus J, Schulz-Ertner D, Schad L, Essig M, Rhein B, Thillmann CO, Wannenmacher M (2000) Stereotactic fractionated radiotherapy for chordomas and chondrosarcomas of the skull base. Int J Radiat Oncol Biol Phys 47: 591-596

9. Eufinger $\mathrm{H}$, Wehmoller M (2002) Microsurgical tissue transfer and individual computer-aided designed and manufactured prefabricated titanium implants for complex craniofacial reconstruction. Scand J Plast Reconstr Surg Hand Surg 36: 326331

10. Evans HL, Ayala AG, Romsdahl MM (1977) Prognostic factors in chondrosarcoma of bone: a clinicopathologic analysis with emphasis on histologic grading. Cancer 40: 818-831 
11. Ewing $J$ (1939) A review of the classification of bone tumors. Surg Gynecol Ostet 68: 971-976

12. Finn DG, Goepfert H, Batsakis JG (1984) Chondrosarcoma of the head and neck. Laryngoscope 94 : 1539-1544

13. Graham MD, Satal off RT, Kemink JL, Wolf GT, McGillicuddy JE (1984) Total en bloc resection of the temporal bone and carotid artery for malignant tumors of the ear and temporal bone. Laryngoscope 94: 528-533

14. Harsh GR, Ojemann RG (2003) Surgical considerations. In: Harsh G, Ojemann R, Janecka IP, Mankin HJ, Suit HD (eds) Chordomas and chondrosarcomas of the skull base and spine. Thieme, New York Stuttgart, $\mathrm{p}$ 80-97

15. Harwood AR, Krajbich Jl, Fornasier VL (1980) Radiotherapy of chondrosarcoma of bone. Cancer 45 2769-2777

16. Hassounah M, Al-Mefty O, Akhtar M, Jinkins JR, Fox JL (1985) Primary cranial and intracranial chondrosarcoma. A survey. Acta Neurochir 78: 123-132

17. Hug EB, Slater JD (2000) Proton radiation therapy for chordomas and chondrosarcomas of the skull base. Neurosurg Clin North Am 11: 627-638

18. Koch BB, Karnell LH, Hoffman HT, Apostolakis LW, Robinson RA, Zhen W, Menck HR (2000) National cancer database report on chondrosarcoma of the head and neck. Head Neck 22: 408-425

19. Korten $A G$, ter Berg $H J$, Spincemaille $G H$, van der Laan RT, van de Wel AM (1988) Intracranial chondrosarcoma: review of the literature and report of 15 cases. J Neurol Neurosurg Psych 65: 88-92

20. Krömer JH, Ludwig K, Bürger H, Alberty J (2002) Chondrosarkom des Siebbeins und der Keilbeinhöhle. Ein Fallbericht. Laryngorhinootologie 81: 702-705

21. Kveton JF, Brackmann DE, Glasscock ME, House WF, Hitselberger WE (1986) Chondrosarcoma of the skull base. Otolaryngol Head Neck Surg 94: 23-32

22. Lau DP, Wharton SB, Antoun NM, Bottrill ID, Moffat DA (1997) Chondrosarcoma of the petrous apex. Dilemmas in diagnosis and treatment. J Laryngol Otol 111:368-371

23. Meyers SP, Hirsch WL, Curtin HD, Barnes L, Sekhar LN, Sen C (1992) Chondrosarcomas of the skull base: MR imaging features. Radiology 184: 103-108

24. Muthukumar N, Kondziolka D, Lunsford LD, Flickinger JC (1998) Stereotactic radiosurgery for chordoma and chondrosarcoma: further experiences. Int $J$ Rad Oncol Biol Phys 41: 387-392

25. Nakayama B, Hasegawa Y, Hyodo I, Ogawa T, Fujimoto Y, Kitano H, Torii S (2004) Reconstruction using a three-dimensional orbitozygomatic skeletal model of titanium mesh plate and soft-tissue free flap transfer following total maxillectomy. Plast Reconst Surg 114: 631-639

26. Neff B, Sataloff RT, Storey L, Hawkshaw M, Spiegel JR (2002) Chondrosarcoma of the skull base. Laryngoscope 112: 134-139

27. Noel G, Habrand JL, Mammar H et al. (2001) Combination of photon and proton radiation therapy for chordomas and chondrosarcomas of the skull base: the Centre de Protontherapie D'Orsay experience. Int J Radiat Oncol Biol Phys 51: 392-398

28. Rosenberg AE, Nielsen GP, Keel SB, Renard LG, Fitzek MM, Munzenrider JE, Liebsch NJ (1999) Chondrosarcoma of the base of the skull: a clinicopathologic study of 200 cases with emphasis on its distinction from chordoma. Am J Surg Pathol 23: 1370-1378

29. Sataloff RT, Myers DL, Spiegel JR, Roberts BR, Telian $S$ (1988) Total temporal bone resection for osteogenic sarcoma. ENT J 67: 626-634
30. Schade G, Grundmann T, Jäkel KT, Krupsky G, Leuwer R, Hess M (2002) Langsam progrediente Dysphonie, Dyspnoe, Dysphagie und Globusgefühl. HNO 50: 1012-1013

31. Schipper J, Maier W, Arapakis I, Spetzger U, Laszig R (2004) Navigation as a tool to visualize bone-covered hidden structures in transfrontal approaches. J Laryngol Otol 118: 849-856

32. Schulz-Ertner D, Haberer T, Jakel $O$ et al. (2002) Radiotherapy for chordomas and low-grade chondrosarcomas of the skull base with carbon ions. Int J Radiat Oncol Biol Phys 53: 36-42

33. Seifert G, Donath K (1998) Kraniale und zervikale Chordome. Ein differentialdiagnostisches Problem. Mund Kiefer Gesichtschir 2: 153-159

34. Sen CN, Sekhar LN, Schramm VL, Janecka IP (1989) Chordoma and chondrosarcoma of the cranial base: an 8-year experience. Neurosurg 25: 931-941

35. Stapleton SR, Wilkins PR, Archer DJ, Uttley D (1993) Chondrosarcoma of the skull base: a series of eight cases. Neurosurg 32: 348-356

36. Steurer M, Kautzky M, Zrunek M (1993) Ein Chondrosarkom der Nase und der Nasennebenhöhlen. HNO 41: 30-32

37. Strauss G, Bücheler M, Bootz F, Oeken K (1999) Retrolaryngealer Tumor mit subglottischer Stenosierung. HNO 47: 920-921

38. Tachibana E, Saito K, Takahashi M, Fukuta K, Yoshida J (2000) Surgical treatment of a massive chondrosarcoma in the skull base associated with Maffucci's syndrome: a case report. Surg Neurol 54: 165-179 